\title{
MATHEMATICAL MODELS FOR ACOUSTIC SPECTRA SIMULATION
}

The experimentally obtained acoustic spectra of some investigated materials; particularly the ion conductive glasses and MOS (metaloxide-semiconductor) structures are analyzed using suitable theoretical models and mathematical procedure to fit the experimental data. The acoustic spectra of ion conductive glasses can reflect the basic features of the relaxation and transport processes of the mobile ions. The results obtained form acoustic deep level transient spectroscopy (A-DLTS) spectra of MOS structures are used for the characterization of deep centers and determination of some of their physical parameters. Suitable theoretical model and mathematical description of acoustic spectra are necessary to understand both the principle of the ionic hopping motion and relaxation processes connected with the mobility of conductive ions including the role of their composition and the distribution of interface traps.

\section{Introduction}

A considerable interest is given to experimental study of glassy materials with the fast ion transport because they play an important role in a number of modern electrochemical devices such as solidstate batteries (portable batteries for heart pacemakers, mobile telephones and laptop computers), solid-oxide fuel cells, electrochemical sensors, electrochronic displays and oxygen-separation membranes [1].

The acoustic attenuation measurement seems to be a useful technique for nondestructive investigation of transport mechanisms in conductive glasses and compared to the electrical ones it has even some advantages as high sensitivity, the absence of contact phenomena and so on $[1,2]$. Acoustical measurements made over a wide range of frequencies and temperatures can characterize different relaxation processes according to corresponding transport mechanisms due to the strong acousto-ionic interaction. In glassy electrolytes, the mobile ions encounter different kinds of site and ionic hopping motion and relaxation processes connected with charge mobility so that the modified jump relaxation model [3] connected with the genetic algorithm [4] can be used for transport mechanisms description.

The interface states in MOS structures have been investigated for more than thirty years using many useful experimental techniques. One of the most important methods is the deep level transient spectroscopy (DLTS) originally developed in 1974 [5] with some modification, one of them is also an acoustic version (ADLTS) [6, 7]. The A-DLTS version is the high-frequency ultrasonic method based on analysis of acoustoelectric response signal (ARS) of the MOS structure when longitudinal acoustic waves propagate through a structure after applied bias voltage steps to the structure. The ARS reflects relaxation processes in both semiconductor and interface layers. It was namely found that the acoustoelectric signal produced by MOS structures reflects any changes in the space charge distribution due to the external condition changes [7].

In this contribution we present theoretical mathematical models for description of experimental results obtained from acoustic spectra of a set of glasses prepared in the system $\mathrm{CuI}-\mathrm{CuBr}-\mathrm{Cu}_{2} \mathrm{O}$ $\mathrm{P}_{2} \mathrm{O}_{5}$ with the purpose to study ion transport mechanisms and to find the role of cuprous halides producing $\mathrm{Cu}^{+}$ions. Another theoretical model is presented for the characterization of the acoustic spectra of MOS structure in the case when the A-DLTS spectra are weak and we cannot analyze Arrhenius plots. Using the simulation of one spectrum is necessary to find the activation energies and some other parameters of traps at the insulator - semiconductor interface in MOS structures.

\section{Theoretical models}

\section{a) Ion conductive glasses}

The formal theory of all relaxation processes looks similar. In dilute system containing a low concentration of mobile ions the acoustic attenuation spectrum may be described as a Debye-like, single relaxation time process in which the individual ion hops occur independently of each other. In such cases, the attenuation $\alpha$ for a wave of angular frequency $\omega$ takes the form [8]

$$
\alpha=\Delta\left(\frac{\omega^{2} \tau}{1+\omega^{2} \tau^{2}}\right)
$$

\footnotetext{
* Peter Hockicko ${ }^{1}$, Peter Bury ${ }^{1}$, Peter Sidor ${ }^{1}$, Stanislav Jurecka ${ }^{2}$, Igor Jamnicky ${ }^{1}$

${ }^{1}$ Department of Physics, Faculty of Electrical Engineering, University of Zilina, Slovakia

${ }^{2}$ Department of Engineering Fundamentals, Faculty of Electrical Engineering, University of Zilina, Slovakia
} 
where the parameter $\Delta$ is the relaxation strength and it determines the magnitude of the attenuation peak. It is related to the strain dependence of the mobile ion site energy, or deformation potential $B$ by

$$
\Delta=N B^{2} /\left(4 \pi \rho v^{3} k_{B} T\right),
$$

in which $N$ is the number of mobile ions, $v$ the velocity of the acoustic wave, $\rho$ the density of the solid, $T$ thermodynamic temperature and $k_{B}$ the Boltzmann constant.

The term in the equation (1) in the round brackets describes a Debye peak. The acoustic attenuation will exhibit a maximum when the condition $\omega \tau$ is equal to 1 , where [9]

$$
\tau=\tau_{0} \exp \left(E_{A} / k_{B} T_{\text {peak }}\right)
$$

is the most probable relaxation time, $\omega=2 \pi v, v$ the frequency of acoustic wave. The relaxation processes, described by the Arrhenius equation (3), are characterized by activation energy $E_{A}$ for jumps over the barrier between two potential minima and typical relaxation frequency of ion hopping $1 / \tau_{0} \approx 10^{13}-10^{14} \mathrm{~s}^{-1}$.

In fact all the investigated relaxation peaks are much broader than Debye peak. It can be interpreted as arising from the existence of a distribution of relaxation times due to random deviations in the local arrangement of the system. According to this hypothesis that the relaxation losses are not to large one can write for the acoustic attenuation, in case of a distribution $f(\tau)$ of relaxation times [9],

$$
\alpha=\frac{\Delta}{2 v} \int \frac{\omega^{2} \tau}{1+\omega^{2} \tau^{2}} f(\tau) d \tau .
$$

As a consequence, the $\tau$ distribution can be connected with a distribution of activation energies $E$, representing the heights of the barriers that the ions must surmount to go into the near allowed positions. A useful form of equation (4) that takes into account only $E$ distribution can be derived by the microscopic theory of Jäckle et al. [9]

$$
\alpha=\frac{B^{2}}{4 \pi \rho \nu^{3} k_{B} T} \int P(E) \frac{\omega^{2} \tau(E)}{1+\omega^{2} \tau^{2}(E)} d E,
$$

where an average deformation potential $B$ expresses the coupling between the ultrasonic stress and the system and $P(E)$ represents the $E$ distribution function. We can assume for $P(E)$ a Gaussian distribution [10]

$$
P(E)=\frac{N}{\sqrt{2 \pi E_{0}^{2}}} \exp \left[-\frac{\left(E-E_{m}\right)^{2}}{2 E_{0}^{2}}\right],
$$

in which $N$ is the total number of jumping particles per unit volume, $E_{m}$ the most probable activation energy and $E_{0}$ the width of the distribution.

This approach depends on the assumption that ion migration may be treated in terms of a set of non-interacting Debye-like processes. However, in solid electrolytes the mobile ions concentrations are large and conduction mechanisms are thought to be cooperative. The relaxation phenomena observed in a wide variety of materials exhibit a power-law type of frequency dependence. The relationship to Debye behaviour is expressed in the form [8]

$$
\alpha \approx \frac{1}{T}\left(\frac{(\omega \tau)^{m}}{1+(\omega \tau)^{1+m+n}}\right),
$$

where $m$ and $n$ are power-law exponents, which take values between 0 and 1 . When $m=1$ and $n=0$, equation (7) reduces to the equation for a single Debye-like process.

Two functions have mainly been used to fit mechanical loss data [11]. The first function is the Kohlrausch-Williams-Watts (KWW) function

$$
\Phi(t, T)=\exp \left[-\left(\frac{t}{T}\right)^{\beta}\right]
$$

with $0<\beta \leq 1$. The acoustical attenuation is then given by

$$
\alpha(\omega, T) \propto \int_{0}^{\infty}\left(-\frac{d \Phi(t)}{d t}\right) \sin (\omega t) d t .
$$

The second function is the double power law (DPL)

$$
\alpha(\omega, T) \propto \frac{1}{(\omega \tau)^{-n}+(\omega \tau)^{m}} .
$$

Using these functions, we can fit also the acoustic attenuation spectrum of the cuprous halides glasses. All glasses we studied using acoustic spectroscopy exhibit an Arrhenius - type relaxation between the peak temperature and the applied frequency

$$
v=v_{0} \exp \left(-\frac{E_{A}}{k_{B} T_{\text {peak }}}\right),
$$

where $v$ is the frequency, $v_{0}$ the preexponential factor, $T_{\text {peak }}$ the temperature of peak maximum which can be easily and directly determined from theoretical fits.

\section{b) MOS structures}

The basic principle of A-DLTS technique consists in utilization of the ARS produced by MOS structure interface when a high frequency longitudinal acoustic wave traverses the structure. After an injection pulse has been applied to the semiconductor structure the amplitude of ARS follows the accumulated charge behaviour over the capacitance, so that the ARS is proportional to the nonequilibrium carrier density $\Delta n$ [7]

$$
\Delta n(t)=\Delta n_{t_{0}} \exp \left(-\frac{t}{\tau}\right),
$$

where $\Delta n_{t_{0}}$ represents the variation in trap occupancy due to the acoustoelectric effect and $\tau$ is the time constant (relaxation time) associated with the release of the carrier from deep centers.

The measured acoustoelectric response signal amplitude for the discrete level can be then given by

$$
U_{a c}^{0}(t)=U_{0} \exp \left(-\frac{t}{\tau}\right)
$$


Including the regime of small-signal excitation, the A-DLTS output signal for the rate window $(R W) \Delta t=t_{2}-t_{1}$ takes the form

$$
\Delta U_{a c}^{0}=U_{a c}^{0}\left(t_{1}\right)-U_{a c}^{0}\left(t_{2}\right)=U_{0}\left(\exp \left(-\frac{t_{1}}{\tau}\right)-\exp \left(-\frac{t_{2}}{\tau}\right)\right),(14
$$

where $\tau$ is the time constant of the acoustoelectric response delay. Note that Eq. (14) is accurate if the trap could be refilled to its equilibrium occupancy before each bias voltage step $\Delta U$. Evidently, with the rate window $\Delta t$ and corresponding $\tau_{\max }$ (for the Lang's method $\tau_{\max }=\left(t_{2}-t_{1}\right) / \ln \left(t_{2} / t_{1}\right)$, the $\Delta U_{a c}^{0}(T)$ (14) peak should appear at the temperature $T=T_{\max }$, when the relaxation time is equal to the time constant of emission process $\left(\tau=\tau_{\max }\right)$.

The reciprocal value of $\tau$ gives the emission rate which for electrons can be expressed by the relation

$$
e=\tau^{-1}=\gamma_{n} \sigma_{n} T^{2} \exp \left(-\frac{E_{A}}{k_{B} T}\right)
$$

were $\gamma_{n}$ is a constant, $\sigma_{n}$ the capture cross section, $E_{A}=E_{C}-E_{T}$ the trap activation energy related to the bottom of conduction band.

Using the A-DLTS technique based on the computer-evaluated isothermal transients and correlation procedure with higher order on-line filters and rectangular weighting function [12], the activation energies $E_{A}$ and corresponding capture cross-section $\sigma_{n}$ of traps can be determined from Arrhenius type dependence $\ln \left(\tau_{\max } T_{\max }^{2}\right)$ versus $1 / T_{\max }$.

Another method for determining the basic parameters of traps at the insulator - semiconductor interface is the modelling of measured spectra [13]. With several trapping centers being present, $U_{a c}^{0}(t)$ is composed of corresponding components

$$
U_{a c}^{0}(t)=U_{i 0}+\sum_{j=1}^{n} U_{j 0} \exp \left(-\frac{t}{\tau_{j}}\right),
$$

where $U_{i 0}$ is the instantaneous acoustoelectric response of the device and the second term on the right hand side of Eq. (16) is the excess acoustoelectric response due to charging (discharging) of the $n$ traps which are captured (emitted) by the rate $e_{j}=\tau_{j}^{-1}$. We can consider that only

$$
\tau_{j}=\tau_{j 0} \exp \left(\frac{E_{A j}}{k_{B} T}\right)
$$

undergoes changes due to scanning the device temperature $T$.

Using the Eq. (16) and Eq. (17), respectively Eq. (14) for discrete levels we can fit the measured A-DLTS spectra for the various rate windows $\Delta t$. The activation energies $E_{A}$ determined from this modelled spectra expressed by the relation

$$
\Delta U_{a c}^{0}(T)=\sum_{j=1}^{n} U_{j 0}\left(\exp \left(-\frac{t_{1}}{\tau_{j}}\right)-\exp \left(-\frac{t_{2}}{\tau_{j}}\right)\right)
$$

can be then compared with the activation energies calculated from Arrhenius plots.

\section{Experimental results}

\section{a) Ion conductive glasses}

The preparation of glasses in the investigated system $\mathrm{CuI}$ $\mathrm{CuBr}-\mathrm{Cu}_{2} \mathrm{O}-\mathrm{P}_{2} \mathrm{O}_{5}$ and the measure equipment has been already described [14]. Using the theoretical models we can fit the temperature-dependent acoustic attenuation spectra at constant frequency. It is usually supposed [10] that the double power law (DPL) provides a slightly better fit than the KWW function or Debye-like process.

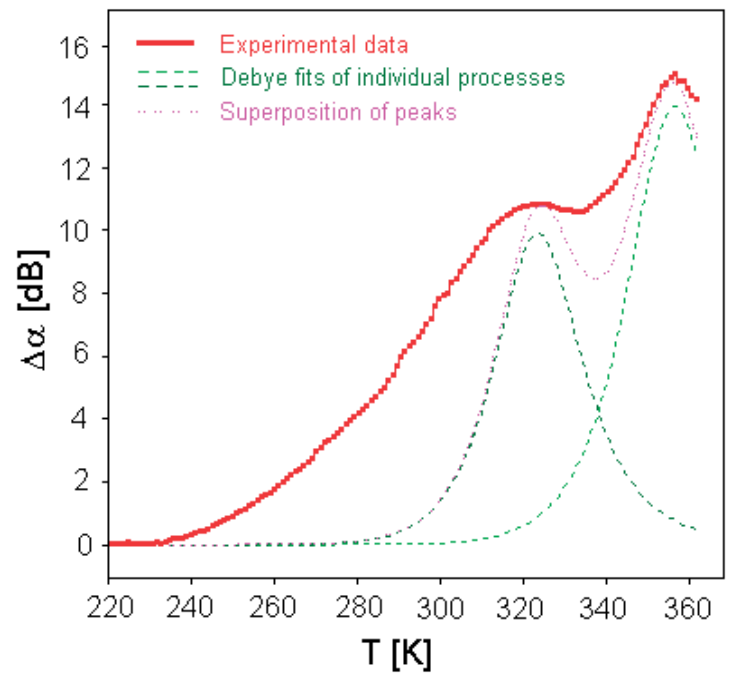

Fig. 1. The acoustic spectrum of glass sample BIDP5 (starting glass composition in mol. \%: 9.09CuI-9.09CuBr-54.55C $\mathrm{Cu}_{2} \mathrm{O}-27.27 \mathrm{P}_{2} \mathrm{O}_{5}$ ) (full line) and the Debye fits of the two relaxation processes (dashed line).

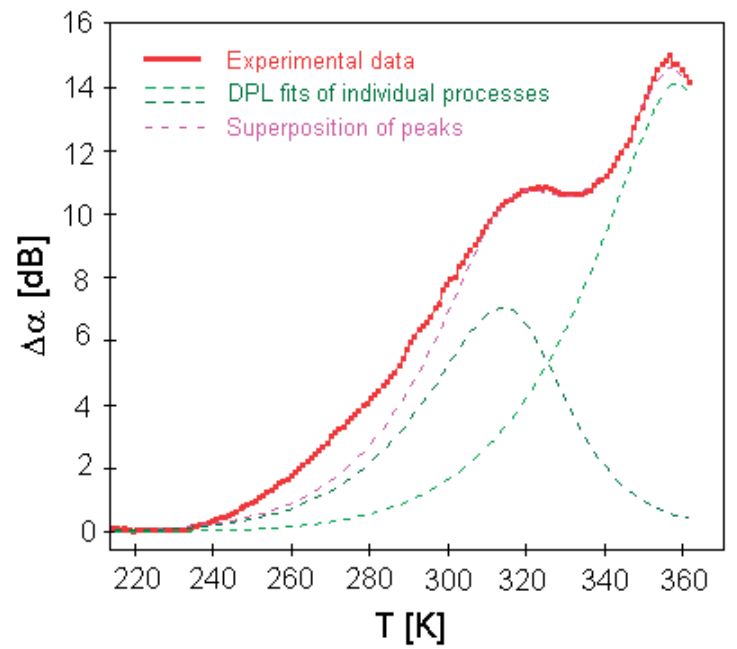

Fig. 2. The acoustic spectrum of glass sample BIDP5 (full line) and the DPL fits of the two supposed relaxation processes (dashed line) 
Firstly, we tried to fit the measured temperature dependence of acoustic attenuation spectra at constant frequency $v=18 \mathrm{MHz}$ using theoretical models (Eq. 1, 7, 8, 10).

The measurements of the temperature dependence of acoustic attenuation indicated in all investigated samples one broad attenuation peak at higher temperature, in which we initially supposed two separated peaks. Debye model (Fig. 1) isn't suitable for modelling relaxation processes in investigated glasses of the system $\mathrm{CuI}-\mathrm{CuBr}-\mathrm{Cu}_{2} \mathrm{O}-\mathrm{P}_{2} \mathrm{O}_{5}$. As we can see, the complete spectrum of sample BIDP5 illustrated in Fig. 2, cannot be fitted supposing only two relaxation processes. Using the theoretical double power law (DPL) model (Eq. 10) we tried to fit the broad attenuation peak as a superposition of three calculated lines represented by the cross-marked line in Fig. 3. The fit gave an excellent agreement with the measured spectrum in high and middle temperature range for the parameters $m=0.27$ and $n=0.42$. We found that the additional third relaxation process should be taken into account with maximum at the temperature around $280 \mathrm{~K}$. The whole temperature dependence of acoustic attenuation was analyzed then assuming the existence of four thermally activated relaxation processes of ions in connection with different kinds of sites. The fourth peak, however, was detected at lower temperatures.

The distinctive peaks of acoustic attenuation spectra, which are caused by resonant interaction with the mobile ion hopping processes, enable us then to study relaxation and transport mechanisms in the ion conductive glasses. The attenuation spectra can be explained by the assumption that temperature peaks are caused by the relaxation processes of mobile $\mathrm{Cu}^{+}$ions in connection with different kinds of sites.

There are several categories of relaxation processes connected with temperature peaks of individual processes. High temperature activation energy presents the basic mechanism. The second mecha-

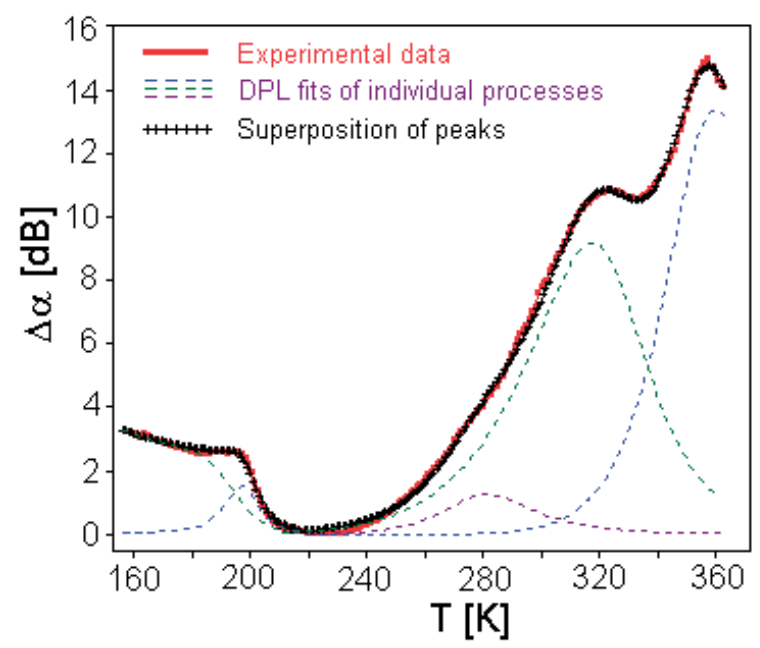

Fig. 3. The acoustic spectrum of glass sample BIDP5 (full line). Crossmarked line represents the best fit of superposition at least four relaxation processes described by DPL model nism observed by both acoustic and conductivity measurements [15] can play an important roll in ionic transport, too. The relaxation mechanisms at the temperature of about $280 \mathrm{~K}$ do not influence the ionic transport significantly. Comparing the activation energies obtained from acoustic and electrical measurements [15], it seems reasonable that essentially the same microscopic processes can be responsible for the acoustic and electrical relaxation processes. However, some differences can be caused by the different relaxation mechanisms connected with ion hopping transport at ac electric field and the hopping of mobile ions due to the interaction of acoustic wave with glass network.

The results from IR spectra of the $\mathrm{CuI}-\mathrm{CuBr}-\mathrm{Cu}_{2} \mathrm{O}-\mathrm{P}_{2} \mathrm{O}_{5}$ glasses [16-18] indicate that the thermal activated processes of $\mathrm{Cu}^{+}$ions determined for all samples of investigated systems can be associated mainly with three different structural units - monomeric orthophosphate $\mathrm{PO}_{4}^{3-}$, low-condensed dimeric diphosphate oxoanions $\mathrm{P}_{2} \mathrm{O}_{7}^{4-}$ and $\mathrm{P}_{3} \mathrm{O}_{10}^{5-}$ structure phosphate anions. The local electric field around the trivalent orthophosphate anion $\mathrm{PO}_{4}^{3-}$ is stronger than the local electric field around dimeric diphosphate oxoanions $\mathrm{P}_{2} \mathrm{O}_{7}^{4-}$ and triphosphate $\mathrm{P}_{3} \mathrm{O}_{10}^{5-}$ anions because the diphosphate and triphosphate anions have smaller negative electric charge on non-bridging oxygen atoms. Moreover, expanded structure of chain groups creates advantageous conditions for ionic motion [18]. Because of this the electrostatic interactions between the mobile $\mathrm{Cu}^{+}$ions and orthophosphate anions are stronger than those between $\mathrm{Cu}^{+}$ions and diphosphate and triphosphate anions and we can suppose that the relaxation processes with biggest activation energies can be connected with monomeric orthophosphate anions and the processes with smaller energies can be connected with both dominant diphosphate oxoanions $\mathrm{P}_{2} \mathrm{O}_{7}^{4-}$ and low condensed triphosphate anions and maybe other polymeric structural units.

\section{b) MOS structures}

The investigated $\mathrm{Al}_{-} \mathrm{SiO}_{2}$-Si MOS structure was fabricated on n-type Si substrates with (100) surface orientation and $1-20 \Omega \mathrm{cm}$ resistivity. The aluminium electrodes were deposited on oxide layer of the thickness of $4 \mathrm{~nm}$. All characteristics were obtained at the temperature range $160-360 \mathrm{~K}$. The basic data were received from sampled acoustoelectric isothermal transients $U_{a c}(t)$ in response to a bias voltage step $\Delta U$ at point $t_{1}$ and $t_{2}=2 t_{1}$ (or $t_{3}=4 t_{1}$, $\left.t_{4}=8 t_{1}, \ldots, t_{n}=2^{n-1} t_{1}\right)$ respectively $\left(t_{1}=0.015 \mathrm{~s}, t_{2}=0.030 \mathrm{~s}\right.$, $t_{3}=0.060 \mathrm{~s}, t_{4}=0.120 \mathrm{~s}, t_{5}=0.240 \mathrm{~s}, t_{6}=0.480 \mathrm{~s}, t_{7}=0.960 \mathrm{~s}$ and $\left.t_{8}=1.92 \mathrm{~s}\right)$.

Fig. 4 represents the typical ARS transients measured at various temperatures and the same bias voltage $U_{g}=-0.2 \mathrm{~V}(\Delta U=0.5 \mathrm{~V})$ for investigated MOS structure.

Fig. 5 shows the series of A-DLTS spectrum determined from the isothermal transients for various time constants. The obtained activation energy calculated from Arrhenius plot (Fig. 6) related to the bottom of conduction band $E_{A}=E_{C}-E_{T}=(0.37+/-0.01)$ $\mathrm{eV}$ with the cross section $5.2 \times 10^{-17} \mathrm{~cm}^{2}$ corresponding to the bias voltage $U_{g}=-0.2 \mathrm{~V}$. 


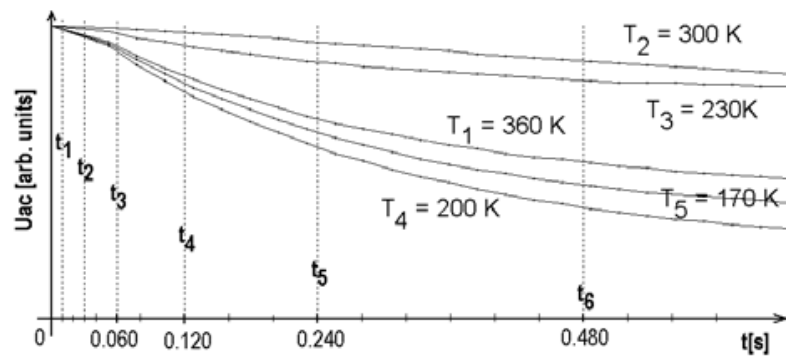

Fig. 4. The ARS transients at various temperatures: $T_{1}=360 \mathrm{~K}, T_{2}=$ $300 \mathrm{~K}, T_{3}=230 \mathrm{~K}, T_{4}=200 \mathrm{~K}$ and $T_{5}=170 \mathrm{~K}$

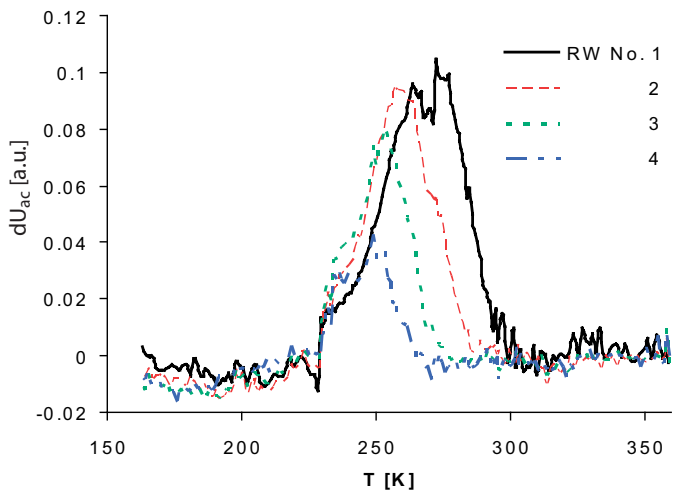

Fig. 5. A set of A-DLTS spectra measured at $U_{g}=-0.2 \mathrm{~V}$, $\Delta U=0.5 \mathrm{~V}$ calculated from isothermal transients for the relaxation times $9.13 \mathrm{~ms}$ (rate window No. (1), $18.4 \mathrm{~ms}$ (2), $36.8 \mathrm{~ms}$ (3) and $73.6 \mathrm{~ms}(4)$

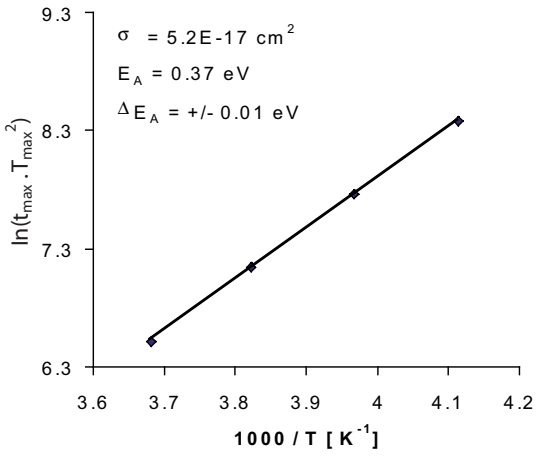

Fig. 6. Arrhenius plot constructed from the positions of the peak maxima of the A-DLTS spectra

From the A-DLTS spectra (Fig. 5) for various relaxation times we also tried to find some information about traps at the insulator-semiconductor interface applying the mathematical methods of simulation. For this reason it was important to corre- late these measured results via exponential analysis. Using the Eq. (18) for the discrete level we simulated the main peak of measured spectra (Fig. 5) for the point $t_{1}=15 \mathrm{~ms}$ and $t_{2}=30 \mathrm{~ms}$ (Fig. 7). We found the energy level $E_{A 1}^{\prime}=0.39 \mathrm{eV}$, which is very close to the activation energy calculated from Arrhenius plot. But using a modelling method of the whole measured spectra we could find next possible trap with energy level $E_{A 2}^{\prime}=0.48 \mathrm{eV}$.

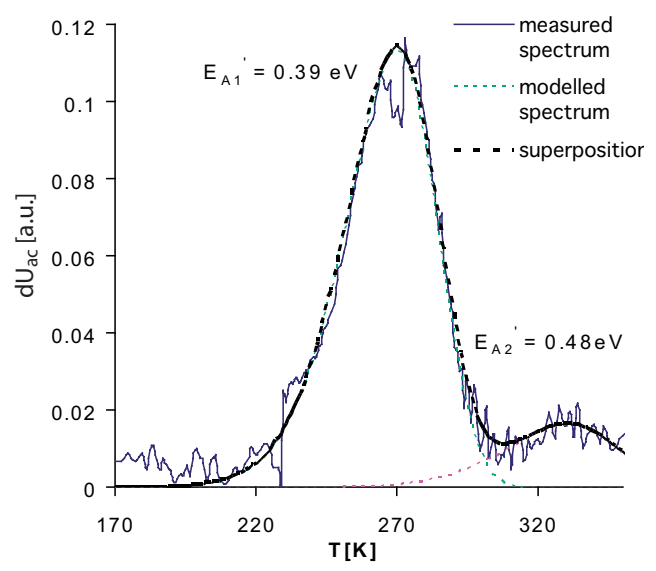

Fig. 7. The simulation peaks of A-DLTS spectra obtained for the relaxation time $9.13 \mathrm{~ms}$ and their superposition

\section{Conclusion}

The experimental and theoretical investigation of ion conductive glasses in system $\mathrm{CuI}-\mathrm{CuBr}-\mathrm{Cu}_{2} \mathrm{O}-\mathrm{P}_{2} \mathrm{O}_{5}$ proved that acoustical spectroscopy connected with suitable mathematical modelling can be a very useful technique for the study of mechanisms in fast ion conductive glasses. Using the theoretical mathematical models of the relaxation processes and simulation of acoustic spectra we can better determine and describe the transport mechanism of mobile ions. Fitting the whole acoustic spectra as a superposition of individual relaxation processes helped us to find additional relaxation process, which could not be found directly from the position of peaks of temperature dependence of acoustic attenuation of ion conductive glasses or electrical measurements.

The A-DLTS measurement technique connected with simulation of acoustic spectra seems to be an effective method for the investigation of the trap at the insulator-semiconductor interface in MOS structures, too. Applying simulation of A-DLTS spectra we identified the similar activation energy of the deep centers at $\mathrm{SiO}_{2}$-Si interface as we calculated from Arrhenius plot. But utilizing the method of simulation of acoustic spectra we could find also next possible traps.

Using other improved mathematical models connected with several mathematical correlation methods should help us to find other useful information about both relaxation or transport 
processes in ion conductive glasses and interface states in MOS structures.

\section{Acknowledgements}

The authors would like to thank assoc. prof. M. Jamnicky from the Department of Ceramic, Glass and Cement, Slovak Technical University, Bratislava for preparation and provision of investigated ion conductive glasses, prof. H. Kobayashi, assoc. prof. M. Takahashi and Dr. K. Imamura from the Institute of Scientific and Industrial Research, Osaka University, Japan for preparation and provision of an investigated MOS sample and Mr. F. Cernobila for technical assistance. This work was supported by VEGA project No. 2/7120/07 and project APVV-0577-07 of the Ministry of Education of the Slovak Republic.

The authors wish to thank for the support to the R\&D operational program "Centre of excellence of power electronics systems and materials" for their components. The project is funded by the European Community, ERDF - European regional development fund.

\section{References}

[1] ROLING, B., HAPPE, A., INGRAM, M. D., FUNKE, K: J. Phys. Chem. B 103 (1999) 4122

[2] CHARNAZA, E. V., BORISOV, B. F., KULESHOV, A. A.: Proc. World Congress on Ultrasonics, Berlin (1995) 483

[3] FUNKE, K.: Solid State Ionics 94 (1997) 27

[4] BURY, P., HOCKICKO, P., JURECKA, S., JAMNICKY, M.: Physica Status Solidi (c) 11 (2004) 2888

[5] LANG, D. V.: Appl. Phys. 45 (1974) 3023

[6] TABIB-AZAR, M., HAJJAR, F.: IEEE Trans. Electron Dev. 36 (1989) 1189

[7] BURY, P., JAMNICKY, I., DURCEK, J.: Physica Status Solidi (a) 126 (1991) 151

[8] ALMOND, D. P., WEST, A. R.: Solid State Ionics 26 (1988) 265

[9] CARINI, G., CUTRONI, M., FEDERICO, M., GALLI, G., TTIPODO, G.: Physical Review B 30 (1984) 7219

[10] BORJESSON, L.: Physical Review B 36 (1987) 4600

[11] ROLING, B., INGRAM, M. D.: Physical Review B 57 (1998) 14192

[12] BURY, P., JAMNICKY, I., HOCKICKO, P.: Communications No. 2, (2003) 5

[13] PINCIK, E., KOBAYASHI, H., RUSNAK, J., TAKAHASHI, M., BRUNNER, R., JERGEL, M., MORALES-ACEVEDO, A., ORTEGA, L., KAKOS, J.: Applied Surface Science 252 (2006) 7713

[14] HOCKICKO, P., BURY, P., JURECKA, S., JAMNICKY, M., JAMNICKY, I.: Advances in Electrical and Electronic Engineering, Vol. 3, No. 2 (2004) 243

[15] BURY, P., HOCKICKO, P., JAMNICKY, M.: Advanced Materials Research 39-40 (2008) 111

[16] JAMNICKY, M., ZNASIK, P., TUNEGA, D., INGRAM, M. D.: J. Non-Cryst. Solids 185 (1995) 151

[17] ZNASIK, P., JAMNICKY, M.: Solid State Ionics 92 (1996) 145

[18] ZNASIK, P., JAMNICKY, M.: Solid State Ionics 95 (1997) 207 